\title{
Nusinersen as a Therapeutic Agent for Spinal Muscular Atrophy
}

\author{
Qing Li \\ Department of Function, ShiJiaZhuang Traditional Chinese Medical Hospital, ShiJiaZhuang, HeBei, China.
}

\begin{abstract}
The reduction of survival motor neuron (SMN) protein causes spinal muscular atrophy (SMA), an autosomal recessive neuromuscular disease. Nusinersen is an antisense oligonucleotide, approved by the FDA, which specifically binds to the repressor within SMN2 exon 7 to enhance exon 7 inclusion and augment production of functional SMN protein. Nusinersen is the first new oligonucleotide-based drug targeting the central nervous system for the treatment of SMA. This review of nusinersen will discuss its action mechanism, cellular uptake, trafficking mechanisms, and administration approaches to cross the blood-brain barrier. Furthermore, nusinersen clinical trials will be assessed in terms of pharmacokinetics, tolerability and safety, the clinical outcomes of multiple intrathecal doses, and a discussion on the primary and secondary endpoints.
\end{abstract}

Key Words: Nusinersen, survival of motor neuron gene, phosphonothioate antisense oligonucleotides, exon splicing, cellular uptake, blood-brain barrier

\section{INTRODUCTION}

Spinal muscular atrophy (SMA) with progressive muscular weakness and atrophy in respiratory, bulbar, and limb muscles is caused by a loss of motor neurons in the anterior horn of the spinal cord. SMA is an autosomal recessive disease that can be divided into four types based on milestone achievement and onset time. Type IV is onset from adulthood, and the other three types are childhood-onset. The most severe is Type I, which includes approximately $60 \%$ of patients, whereas Type III is relatively mild. The incidence of all four types is approximately one in every 11000 live births, and is therefore one of the most common genetic factors for childhood mortality. ${ }^{1}$

A homozygous deletion or loss function mutation in the survival motor neuron 1 (SMN1) gene causes SMA. SMN1 encodes

Received: November 27, 2019 Revised: January 28, 2020

Accepted: February 19, 2020

Corresponding author: Qing Li, MM, Department of Function, ShiJiaZhuang Traditional Chinese Medical Hospital, No. 233, ZhongShan West Road, ShiJiaZhuang, HeBei 050051, China.

Tel: 86-0311-85511616, Fax: 86-0311-85511699, E-mail: liqingliyang@126.com

-The author has no potential conflicts of interest to disclose.

(C) Copyright: Yonsei University College of Medicine 2020

This is an Open Access article distributed under the terms of the Creative Commons Attribution Non-Commercial License (https://creativecommons.org/licenses/ by-nc/4.0) which permits unrestricted non-commercial use, distribution, and reproduction in any medium, provided the original work is properly cited. a ubiquitously expressed 38-kDa protein, which is mostly localized in the cytoplasm and nuclear gems, and functions as a small nuclear ribonucleoprotein particle (snRNP) for complex assembly with an essential role in cell survival. ${ }^{2,3}$

Survival motor neuron 2 (SMN2) is a nearly identical SMN gene copy with SMN1 in humans. Both genes consist of inverted repeat sequences on chromosome 5q13, different from 11 nucleotides. ${ }^{4}$ Exon 7 skipping during pre-mRNA splicing is caused by a C-to-T substitution at position 6 of exon 7, from SMN1 to SMN2. The C6T transition disrupts an exonic splicing enhancer, which is dependent on splicing factor 2/alternative splicing factor (SF2/ASF) and is present in SMN1 exon 7 as heptamer motif (nucleotides +6 to +13 , CAGACAA). An exonic splicing silencer (ESS) in SMN2 is also created by the C6T transition in which heterogeneous nuclear ribonucleoprotein (hnRNP) Al, a known repressor protein, binds to ESS to inhibit exonic splicing. ${ }^{5}$ Exon 7 is excluded, and an unstable truncated protein is produced in $80-90 \%$ of the transcripts that are rapidly degraded. ${ }^{6}$ Exon 7 skipping results in SMN2 being unable to supplement for the loss of SMN1 and causing SMA.

As a result, correcting exon 7 splicing in SMN2 has becomes an attractive approach for therapy, as all patients with SMA have SMN2. Several strategies have been undertaken to promote the inclusion of SMN2 exon 7. These include using small molecules to amplify SMN2 transcription, ${ }^{7}$ adeno-associated 
virus-mediated gene delivery to replace $\mathrm{SMN},{ }^{8}$ chimeric or bifunctional antisense oligonucleotides (ASOs), or trans-splicing RNAs to block intronic splicing silencers (ISSs)/exonic splicing silencers or junction of the intron 7-exon 8. ${ }^{9,10}$ The beneficial effects of these strategies have been demonstrated using models of SMA.

In 2006, an intron-7 ISS of the SMN2, termed ISS-N1, was discovered by Singh, et al. ${ }^{2}$ in human SMN1/2 genes. ISS-N1 is an intronic cis-element that is located in the 5 ' splicing site, downstream of exon 7, and the non-conserved portion of the SMN final intron 7 in humans. This element is considered an essential component that modulates alternative splicing of SMN exon 7 in a regulatory network that is associated with the pathogenesis of SMA. ISS-N1 functions to recruit normally spliced repressors hnRNP A1 and A2 that inhibit exon 7 inclusion.

Nusinersen was discovered by Adrian Krainer and team in 2010. ${ }^{1}$ An 18-mer 2'-O-(2-methoxyethyl) (MOE) phosphonothioate oligodeoxyribonucleotides (ODN), also known as ASO-10-27 5'-TCACTTTCATAATGCTGG-3', is a single-stranded DNA that is designed to target an intron 7 site. Nusinersen hybridizes to ISS-N1 by sequence-specific Watson-Crick base pairing to block hnRNP recruitment, which results in the inclusion of SMN2 exon 7 in pre-mRNA splicing and augments full-length SMN2 protein production.

\section{ACTION MECHANISMS}

Delivering oligonucleotides to the central nervous system (CNS) to develop treatments for CNS disorders has some advantages. Oligonucleotides have high target specificity, accessing drug targets that were previously inaccessible, with reduced toxicity and systemic exposure, as well as longer half-life dosing. Oligonucleotides can also offer multiple approaches for precise downregulation of disease-relevant genes, gene upregulation, correcting mutations and splice editing, which are not easily attained by traditional monoclonal antibodies or small-molecules. ${ }^{11}$ Alternatively, oligonucleotide effects are reversible and different from other gene therapies, whereby a large proportion of the problems related to the many permanent alterations is present. An additional advantage is that the development cycle of oligonucleotides is simplified due to their uniform process of chemistry. For these reasons, oligonucleotides have become promising targets. However, the delivery of oligonucleotides to the CNS has had some substantial obstacles. Foremost, oligonucleotides do not readily cross the blood-brain barrier (BBB), ${ }^{12}$ with fewer than $1 \%$ reaching the brain.

Although intrathecal (IT) or intracerebroventricular (ICV) administration methods could improve CNS delivery, ${ }^{13}$ they are invasive and may increase burden for patients. To facilitate BBB crossing, intranasal or oral administrations are viable options, and a chemical modification has been developed and tested. Another difficulty is that pharmacodynamic (PD) and pharmacokinetic (PK) properties of oligonucleotides are unconventional and remain less studied, although they have profound implications for dose regimens and clinical effectiveness. Furthermore, the action mechanism of oligonucleotide has yet to be elucidated, and more studies are required to unravel the complex molecular processes.

Phosphonothioate backbone modification was introduced to develop oligonucleotide drugs, ${ }^{14}$ in which one of the oxygens in the phosphate moiety was substituted by sulfur. Nuclease resistance was increased, and a link with carrier proteins in the blood was facilitated by phosphonothioate modification, which led to increased absorption, longer half-life, and slower excretion via the kidneys. ${ }^{13}$ A modification of the sugar moiety in which hydroxyl in the 2' position of the sugar moiety of ODN was substituted by 2' MOE groups have further improved DNA binding affinity and bioavailability, and decreased toxicity and immunostimulatory effects of oligonucleotide drugs. ${ }^{15,16}$

ISS-N1 of the SMN2 comprises of 15 nucleotides (CCAG CATTATGAAAG, +10 to +24$)$. CAGCAT $(+11$ to +16$)$ is the first hnRNP Al motif element, and TGAAAG $(+19$ to +24$)$ is the second. A strong ISS consists of two juxtaposed weak motifs of hnRNP Al to produce a strong splicing silencer. Binding of either of the two hnRNP A1/A2 molecules to the intron 7 ISS and other sites results in accumulative hnRNP A1/A2 spreading along the SMN2 exon 7 and its flanking intron sequences, antagonizing the binding of SR proteins, SF2/ASF, Tra2-b1, and other splicing factors that are vital for recognition and splicing activation of exon $7 .{ }^{17}$ Nusinersen abrogates the binding of hnRNP A1/A2 molecules to the ISS-N1 of intron 7, leading to exon 7 splicing activation and inclusion. ${ }^{18}$

\section{OLIGONUCLEOTIDE UPTAKE AND TRAFFICKING IN CELLS}

Currently, cellular uptake and distribution of phosphonothioate antisense oligonucleotides (PS-ASOs) are poorly understood, and have only recently been investigated with limited information on neuronal uptake in vivo. It has been proposed that PS-ASOs enter cells via two pathways, ${ }^{19}$ the productive and non-productive pathways (Fig. 1). Productive pathway guides PS-ASOs to access their targets, and non-productive pathway sequesters PS-ASOs in saturable sinks. The two pathways compete with each other for oligonucleotides. It has been suggested that the ratio for productive uptake of total ODNs to enter into liver tissue is $<20 \% .{ }^{20}$ It is likely that many different non-productive proteins exist, with the candidates including VPS28 and TSG101, ${ }^{21} \mathrm{Ku} 70$ and Ku80 proteins, ${ }^{22}$ the components of ESCRT-I complex (endosomal sorting complexes required for transport), alpha 2 macroglobulin (A2M), ${ }^{23}$ and paraspeckle proteins (including PSPC1, PSF, hnRNPK, and p54nrb). ${ }^{24}$ It is possible that the productive pathway is composed of the following candidate proteins: TCP1 (including 


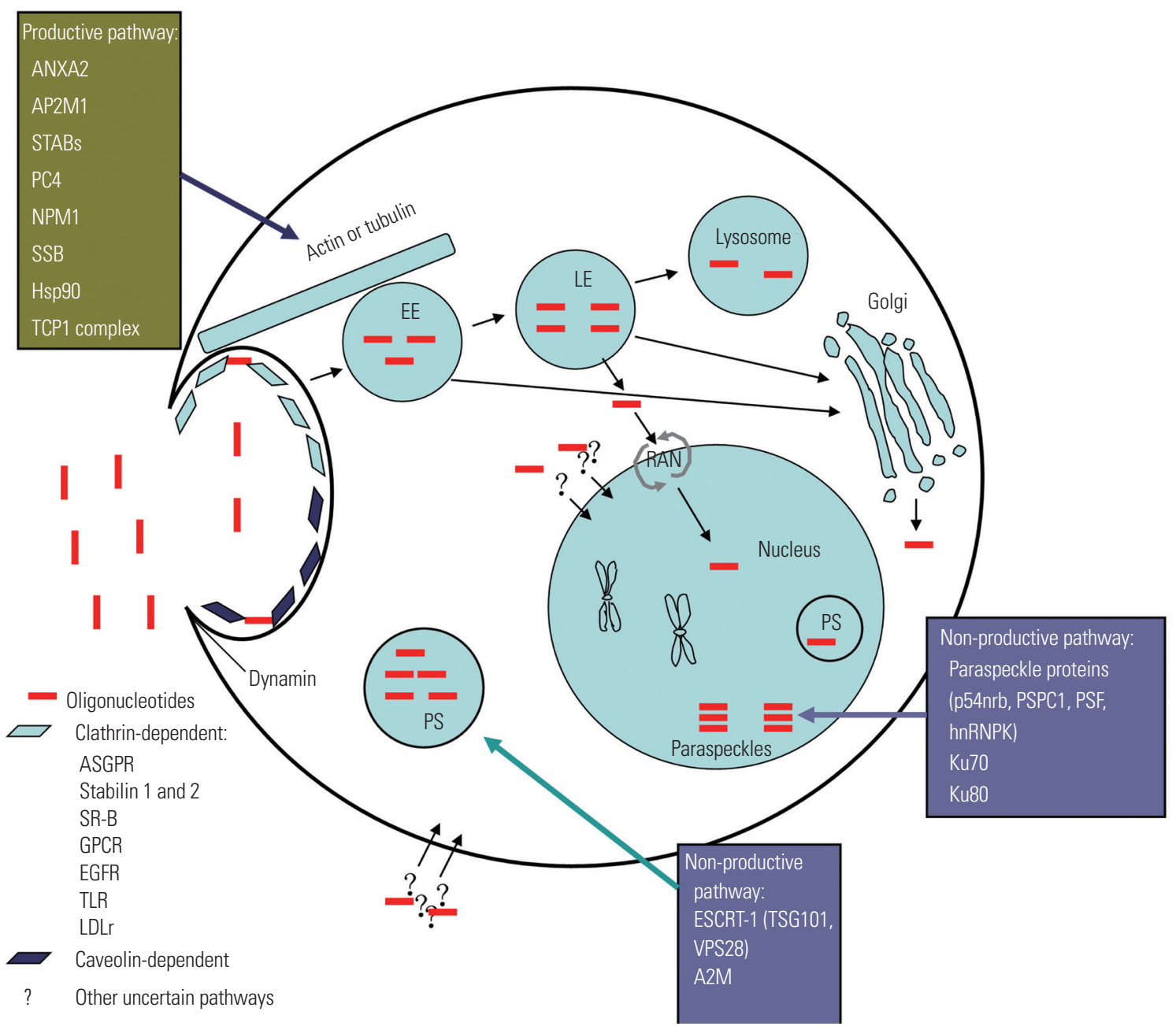

Fig. 1. Oligonucleotide uptake and trafficking in cells. It has been proposed that phosphonothioate antisense oligonucleotides (PS-ASOs) enter cells via two pathways: productive pathway and non-productive pathway. Productive pathway guides PS-ASOs to access their targets (yellow outlines), and nonproductive pathways sequester PS-ASOs in saturable sinks (blue outlines). Uptake and internalization of PS-ASOs can be directed by cell-surface proteins (including receptors) through caveolin- or clathrin-dependent pathways or other pathways of endocytosis. The endocytosis vesicle bud from the membrane and the scission of the plasma membrane is catalyzed by GTPase dynamin. Freed cargo-containing vesicles with PS-ASOs fuse with early endosomes (EE) by being transported along the cytoskeletal structures of tubulin or actin. Internalized ASOs can be transported from EE to late endosomes (LE) and to lysosomes. PS-ASOs predominately accumulate in punctate structures in EE, LE, lysosomes (possibly equal to phosphonothioate bodies of cytoplasm), and Golgi-58K-related vesicles, in which non-productive and productive pathways of uptake may diverge and the majority of oligonucleotides are likely reserved in non-productive pathway. A small number of PS-ASOs can be released from endosomal organelles and escape into productive pathway, involving entry into the nucleus and cytosol to act on target DNA. Entering into the nucleus is likely via the RAN-mediated pathway. PS-ASOs may be linked to paraspeckle proteins in the nucleus and shape functional paraspeckle-like structures.

tubulin, actin, histone deacetylases, and cyclin E1), ${ }^{25} \mathrm{Hsp} 90$, La (also known as SSB), NPM1, AP2M1, ANXA2, ${ }^{26}$ PC4, STAB1, and STAB2 (stabilin family of scavenger receptors). ${ }^{27}$

\section{UPTAKE AND INTERNALIZATION OF PS-ASOs BY MAMMALIAN CELLS}

Two steps illustrate the cellular uptake process: adsorption and internalization. Adsorption does not require energy and can be saturated. ${ }^{28,29}$ PS-ASOs interact with different cell surface proteins (including receptors), either via conjugated ligands or directly. ${ }^{30}$ Once bound to the cell surface, by the di- rection of these proteins, associated ASOs are internalized to enter the cell via caveolin- or clathrin-dependent or via nonconventional endocytic pathways that are generally regarded as productive pathways. ${ }^{31,32}$ Other endocytic pathways may play a role, such as macropinocytosis, ${ }^{33}$ which are generally regarded as non-productive pathways. Several different receptors connected to PS-ASOs uptake are included in clathrin-dependent cell surface proteins, such as GPCR, ${ }^{34}$ SCARA, ${ }^{35}$ ASGPR, SR-B, EGFR, TLR ${ }^{36}$ and LDLr. Currently, integrins are the only caveolin-dependent pathways that have been discovered ${ }^{37}$ It is believe that the cell uptake of PS-ASOs, not using transfection reagents or electroporation, is due to the fact that most internalized ASOs are taken up by pathways in which they can bind 
RNA at given sites and do not result in ASO accumulation. ${ }^{19}$ It is also possible that different cell types express different cell surface proteins, and the productive or non-productive pathway routes could be contributed to ASOs. ${ }^{38}$ For example, DU145 cancer cells could be entered by nucleolin via macropinocytosis, while the nonmalignant skin fibroblast HS27 cells could not. ${ }^{39}$ Some membrane proteins, including SCARA ${ }^{40}$ and nucleolin, have been shown to mediate functional uptake of splicing-modulating PS-ASOs, and the activity of splicing-modulation ASOs have been increased by the use of nucleolin-specific aptamer-mediated delivery of ASOs in different cancer cells. ${ }^{41}$

\section{PS-ASO INTRACELLULAR TRAFFICKING AND BINDING TO A SUBSET OF INTRACELLULAR PROTEINS}

The endocytic vesicle bud from the membrane and plasma membrane scission is catalyzed by GTPase dynamin. Freed cargo-containing vesicles with PS-ASOs fuse with early endosomes (EEs) by being transported along cytoskeletal structures (tubulin or actin). Maturation is undergone by the resulting fusion vesicles. ${ }^{21}$ Transport vesicles eventually fuse with mature late endosome (LE) to form lysosomes by budding from the trans-Golgi network.

ESCRT-I complex (TSG101 and VPS28) allows membrane budding, directs PS-ASOs to non-productive pathways, ${ }^{21}$ and mediates the intraluminal vesicle (ILV) formation during endosome maturation. PS-ASO productive transport from EE to LE and to lysosomes may be facilitated by proteins (AP2M1 and ANXA2). AP2M1 is an adaptor protein in the uptake and distribution process of caveolin- and clathrin-independent productive endocytic pathways. ${ }^{20}$ Trafficking of PS-ASOs is facilitated by the ANXA2 protein from EEs to LEs, and ANXA2 can be recruited to the LE membrane when the presence of PS-ASOs load alters the lipid signature of this organelle. PS-ASOs can also be co-transported with ANXA2, likely in the form of ILVs inside the LE lumen, and ASOs released from LEs may also be facilitated. ${ }^{42}$ Additionally, ASOs can also conjugate with $\mathrm{N}$-acetyl galactosamine (GalNac) to enter cells by the interaction of GalNac with ASGPR receptor via clathrin-mediated endocytosis. ${ }^{43}$ ASGPR and PS-ASOs dissociate in EE, ASGPR receptor is returned to the plasma membrane through recycling, and PS-ASO arrives in the cytosol to target RNA.

Internalized PS-ASOs reach their DNA/RNA targets and bind multiple proteins in the cytosol or nucleus after they are released from liposomes or membrane-enclosed organelles. Using the competition binding assay, Liang and collegues ${ }^{24,26}$ have identified or characterized a total of 58 PS-ASO binding proteins. Several of the binding proteins are chaperones, and many proteins contain nucleic-acid binding domains. Although it has been shown that some proteins do reduce or enhance the activity of PS-ASOs, many have no impact on antisense activi- ties of PS-ASOs. Reduced activities of PS-ASOs by several proteins comes from the fact that several proteins compete with RNase $\mathrm{H} 1$ for binding to the duplex formed between PS-ASOs and their target DNA. Increased activity of some PS-ASO chemistries has been linked to the enhancement of Hsp90 protein levels. Additionally, the retention of PS-ASOs in the nucleus may be increased by NPM1 and La (also known as SSB) proteins, which can thus facilitate PS-ASO activity. La is a chaperone protein required for RNA-binding, processing, and transcription of transfer RNA. NPM1 is a nucleic acid-binding protein involved in the structure of nucleolar ribonucleoproteins. The paraspeckle protein p54nrb and the helicase proteins Ku70 and Ku80, known as nucleic acid binding proteins, were identified to compete with RNase $\mathrm{H}$ for binding to the ASO-DNA duplex to inhibit PS-ASOs activity.

Hsp90 deletion mutations indicate that if the ASO hydrophobic 2' modification moiety is increased, then the protein binding becomes more promiscuous, and the affinity of the protein of ASO is also greater. PS-ASOs with more hydrophobic 2 ' substituents tend to bind to more proteins and with much avidity compared to the hydrophilic 2' MOE PS-ASOs. ${ }^{24,44}$

\section{SUBCELLULAR ACCUMULATION, DISTRIBUTION, AND LOCALIZATION OF TRANSFECTED PS-ASOs}

PS-ASO pathways of free uptake (without a delivery agent) may be different from that of liposome-mediated delivery (transfection). After free uptake, PS-ASOs dominantly accumulate in punctate structures in EEs, LEs, lysosomes (possibly equals to phosphonothioate bodies of cytoplasm), perinuclear structures, P-bodies, and Golgi-58K-related vesicles in the cytoplasm. ${ }^{45}$ After free uptake, although PS-ASOs nuclear accumulations are not directly observed, nuclear activity of ASOs have been reported. Cells have the ability to take up PS-ASOs, and due to this reason the nucleus can accumulate sufficient PS-ASOs to support pharmacological activity. After transfection, ODNs localize and accumulate primarily in the nuclear phosphonothioate bodies (PS bodies, correspond to nuclear punctate sites) ${ }^{24,25,46}$ In addition to PS bodies, other filament and punctate nuclear structures, such as paraspeckles or paraspeckle-like structures, can accumulate PS-ASOs. Furthermore, transfected PS-ASOs in the cytoplasm also form many distinct punctate structures similar to free uptake. ${ }^{45,47-49}$

It has been suggested that during or after lysosome formation, non-productive and productive uptake pathways may diverge. Although a large majority of PS-ASOs accumulate and are maintained in the non-productive pathway (likely in endosomes/lysosomes), and a small number of PS-ASOs can be released from the liposomes or membrane-enclosed organelles and escape into the productive pathway, which probably involves entry into the nucleus and cytosol to act on target DNA..$^{50}$ 
It has been speculated that some unidentified cellular receptors or cargo molecules of transporter systems, shuttled between the nucleus and cytoplasm in part, can be bound by PS-ASOs through a small GTPase known as the RAN-mediated pathway. ${ }^{51}$ The interaction between the members of superfamily of transport receptors and cargo are regulated by the GTP-bound form of RAN that can serve as a sensor in this course. The protein export can be almost entirely abolished by nuclear injection of the RanT24N mutant protein, presumably due to the inhibition and sequestration of RCCl exchange factor depleting the nuclear RanGTP pool. RCC1 is a factor for RAN nucleotide exchange, and can influence the nucleotide-bound states of RAN.

PS-ASOs co-localize with the chaperone T complex 1 subunit $\beta$ (TCP1 $\beta$ ) in PS bodies. TCP1 $\beta$ binding to PS-ASO and PS body formation is dependent on the concentration of PS-ASO. TCP1 $\beta$ and PS-ASOs are observed co-localized in the cytoplasm when RAN levels are reduced. It is also notable that several members of the TCP1 family co-localize with PS-ASOs in endosome structures in the cytoplasm. Therefore, it is likely that the exit of ASO from cytoplasmic endosomes may be facilitated by the TCP1 family, and that TCP1 $\beta$ can enhance PS-ASO activities by facilitating PS-ASOs nuclear accumulation. ${ }^{25}$

PS-ASOs can accumulate in paraspeckles in the nucleus. Localization of paraspeckles appears to be defined by PS-ASOs binding to specific paraspeckle proteins, including PSPC1, PSF, and p54nrb. When the paraspeckles relocate, PS-ASOs move with proteins in paraspeckles in the same fashion as that observed with PS bodies. ${ }^{24}$ A long noncoding RNA, NEAT1, forms on paraspeckles, and it has been indicated that NEAT1 could be displaced by PS-ASOs and PS-ASOs follow to form functional paraspeckles. PS-ASOs localize to unique sites in the nucleus by binding to specific paraspeckle proteins, which can lead to reverse impacts on PS-ASOs. The two following possibilities cannot be excluded. First, paraspeckle proteins exert an inhibitory effect on PS-ASO activity by competing with RNase H1 in order to bind to duplexes between RNA and PS-ASOs. Second, PS-ASOs are sequestrated by the paraspeckle proteins in nuclear filaments or paraspeckles, which may contribute to the inhibition of PS-ASOs activities. Remarkably, drug bioavailability can be enhanced, and off-target effects can be reduced by recruiting a higher dose of ODNs to the productive pathway. Consequently, the functional activity of ODNs could be increased by endeavoring to illustrate the mechanisms of uptake processes, including the identification of primarily ODN-binding proteins.

\section{ASSISTED DELIVERY AND APPLICATION OF PS-ASOs TO THE CNS}

Endocytosis is the mechanism in which naked (unconjugated) PS-ASOs are taken up into cells, similar to macromolecules. ${ }^{52}$
However, naked double-stranded siRNAs are not capable of escaping across the lipid bilayer barrier of the endosome. In comparison, due to the phosphonothioate backbone hydrophobicity, PS-ASOs take an approach termed gymnosis, an unknown mechanism, to escape endosomes across the lipid bilayer into the cytoplasm and nucleus. ${ }^{53,54}$ Gymnosis is a productive silencing pathway, and has been described as "free uptake" by some studies. Without using any transfection reagent, gymnosis promotes productive oligonucleotide uptake by taking advantage of the properties of normal cell growth in tissue culture. This strong, vigorous approach permits multiple targeted sequence-specific silencing in many cell types in vitro, both at mRNA and protein levels. Stein, et al. ${ }^{53}$ have demonstrated that 6-10 days was optimal for gymnotic delivery in 518A2 cells. It took 3-5 days for maximum Bcl-2 protein and mRNA silencing by a 16-mer PS-ASO, called SPC2996 targeted to the Bcl-2 mRNA, which can also lead to silencing of the targeted gene for no less than 240 consecutive days. Significantly, PS-ASOs entered the cellular cytoplasm in this process without a delivery reagent.

PS-ASOs are not capable of crossing the BBB, despite being readily taken up by brain tissues and having a long tissue halflife. However, it was discovered that extensive distribution of PS-ASOs throughout the brain parenchyma could be achieved by IT administration of PS-ASOs into the cerebrospinal fluid (CSF) of monkeys. In the brain parenchyma, the drug was taken up by neurons and other cell types, and it escaped from endosomes into the cellular cytoplasm and nucleus. ${ }^{55}$ As CSF compromises the activity of negligible nucleases to degrade PS-ASOs, it could be regarded as an environmental protectant. ${ }^{56}$ Moreover, it is an immune-privileged zone; therefore, within this fluid, oligonucleotide stability was increased. ${ }^{57,58}$ In the past few years, many different oligonucleotides have been investigated using alternative administration methods such as IT, ICV, or direct injection into specific sites of the brain of experimental animals. ${ }^{56-59}$ The advantages of this method have led to numerous clinical trials in which ASOs, such as nusinersen, can target the CNS in human brain disorders. ${ }^{18,60,61}$

Following administration via IT or ICV routes, PS-ASOs are quickly absorbed from the injection site into the CSF or the circulation. ${ }^{62}$ After IT administration, the distribution of PS-ASOs from CSF lasts between minutes to hours; subsequently, most are taken up into CNS tissues, and a lesser number are transferred into systemic circulation. ${ }^{63}$ Similar to plasma, the twophase course is included for PS-ASO elimination from CSF. Seven days after a single, IT bolus injection in adult cynomolgus monkeys with a dose of 1,3 , or $7 \mathrm{mg}$ of phosphonothioate 2' MOE ODN, the most significant accumulation of ODNs was observed in small and large cell bodies of the gray matter, in accordance with the uptake by neuronal and glial cells. In contrast, intraperitoneal (IP) bolus injection of the same 2' MOE ODN, the concentration for $50 \%$ of maximal effect $\left(\mathrm{EC}_{50}\right)$ in the liver, was over tenfold higher than that in the brain, likely illus- 
trating a higher ratio of uptake of non-productive to productive in the liver. ${ }^{64}$

Furthermore, after a single ICV bolus injection with a dose of $100 \mu \mathrm{g}$ of 2' MOE ODN to correct SMN2 splicing in murine spinal cord and brain, the pharmacological activity could be prolonged. After dosing, the drug could be maintained for more than 36 weeks and pass complete ODN elimination. Notably, after IP bolus injection, the duration of the pharmacological action of ODN in the liver was slightly shorter, with the duration of correcting minimally SMN2 splicing observed 8 weeks after dosing. In the spinal cord and brain, SMN2 splicing correction 71 days after the end of the $30-\mu \mathrm{g} /$ day ICV infusion was equal to the level observed 2 days after the end of infusion. ODNs with a long half-life in peripheral tissues and an extremely long half-life in the CNS can partially explain their persistent effects (due to its immune privileged status and low nuclease levels). ${ }^{64-67}$ It was speculated that the ICV bolus injection of ODNs was a more effective way of delivery than ICV infusion, based on the $\mathrm{EC}_{50}$. The $\mathrm{EC}_{50}$ of nusinersen was $5.7 \mu \mathrm{g} / \mathrm{g}$ for the brain and $1.6 \mu \mathrm{g} / \mathrm{g}$ for spinal cord tissue in ICV bolus injection, compared to $20.2 \mu \mathrm{g} / \mathrm{g}$ for the brain and $9.3 \mu \mathrm{g} / \mathrm{g}$ for the spinal cord in ICV infusion.

One year later, in a 7-day ICV infusion, nusinersen was measurable in motor neurons. The half-life of ICV-infused 2' MOE ODNs in tissue was 145-191 days in the spinal cord and 71-206 days in the brain. Meanwhile, after IV injection in rodent peripheral tissues, the half-life of 2'-O-methyl (2'-OMe) phosphonothioate ODNs was between 10-65 days. A decreased dose of ODNs could be used in IT administration, based on the fact that there was a slower rate of clearance and degradation as well as a relatively small volume contained in the CNS, leading to the possibility of reduced adverse events. ${ }^{64}$

Over $85 \%$ of phosphonothioate ODNs escape into the systemic circulation and bind to circulating plasma proteins, mostly albumin. ${ }^{68}$ This may lead to the difference between the ODNs relative potency observed in vivo and in vitro. For example, a
2' MOE phosphonothioate ODN showed the same potency as a phosphonothioate morpholino oligonucleotide (PMO) ODN in SMA patient fibroblasts in vitro, and was 3-5 times less potent for the inclusion of SMN2 exon 7 in vivo than PMO ODN. This could attribute to a four- to eightfold higher accumulation of 2' MOE phosphonothioate ODN in CNS tissues compared to that of PMO ODN. ${ }^{64}$

The main degradation product of minimal nuclease cleavage of ODNs in vivo is 3' $n$ - 1 oligonucleotides. Owing to the decreased binding to tissue and plasma proteins, shorter endonucleolytic products are quickly eliminated in urine. The primary route to excrete phosphonothioate ODNs is via the kidneys, mainly involving nuclease degradation products, which is a comparatively slow process. $^{68}$

\section{NUSINERSEN EVALUATION IN PHASE 1 SAFETY STUDIES}

In Phase 1 studies, ${ }^{69}$ a single $5 \mathrm{~mL}$ IT bolus injection of nusinersen was administered to a total of 28 children participants, followed by a standard lumbar puncture (LP) (over 1-3 minutes) at the L3-L4 disc space (Table 1). Nusinersen was assessed in four dose level groups (1, 3, 6, $9 \mathrm{mg})$. For safety and PK, CSF was collected by a second LP on day 8 in the 1,3 , and $6 \mathrm{mg}$ dose groups and day 8 or 29 in the $9 \mathrm{mg}$ dose group. A total of 24 out of 28 participants were enrolled in the long-term extension study for assessment 9-14 months post-dose, using the same evaluations as those performed in the single-dose study. The most frequently observed adverse effects (AEs) were back pain (17.9\% of participants), post-LP headache (21.4\%), and headache (39.3\%). An immunogenic response to nusinersen was not detectable 9-14 months after a single IT dose of nusinersen. ${ }^{69}$ In the same Phase 1 study, more frequent AEs were observed in children with Type 3 SMA, older children, and with the use of a 21- or 22-gauge needle in contrast

Table 1. Summary of Nusinersen Clinical Trials

\begin{tabular}{|c|c|c|c|}
\hline & Phase 1 & Phase 2 & Phase 3 (ENDEAR) \\
\hline Participants (n) & 28 & 20 & 121 \\
\hline Age range & $2-14$ years & 3 weeks to 7 months & $<7$ months \\
\hline Delivery method & Intrathecal bolus injection & Intrathecal injection & Intrathecal injection \\
\hline Dose & $1,3,6,9 \mathrm{mg}$ & $6 \mathrm{mg}$ and $12 \mathrm{mg}$ equivalents & 12 mg equivalents \\
\hline Adverse effects (top 5) & $\begin{array}{l}\text { Headache, post-LP headache, back pain, } \\
\text { pyresia, constipation }\end{array}$ & $\begin{array}{l}\text { Pyresia, respiratory infection, constipation, } \\
\text { vomiting, joint contracture }\end{array}$ & $\begin{array}{l}\text { Pyresia, constipation, upper respiratory tract } \\
\text { infection, pneumonia, respiratory distress }\end{array}$ \\
\hline Pharmacokinetics & $\begin{array}{l}\text { CSF concentrations in the } 9 \mathrm{mg} \text { group } \\
29 \text { days after dose ( } 2.33 \pm 0.928 \mathrm{ng} / \mathrm{mL}) \text {. } \\
\text { The half-life in CSF was } 132-166 \text { days. }\end{array}$ & $\begin{array}{l}\text { CSF drug concentrations were greater than } \\
10 \mu \mathrm{g} / \mathrm{g} 15-168 \text { days after dosing. }\end{array}$ & \\
\hline Clinical outcomes & $\begin{array}{l}\text { Improved HFMSE scores in the } 9 \mathrm{mg} \\
\text { groups post-dose. }\end{array}$ & $\begin{array}{l}\text { An increased improvement in HINE-2 and } \\
\text { CHOP-INTEND test assessments. }\end{array}$ & $\begin{array}{l}\text { A higher percentage of motor-milestone } \\
\text { response for primary endpoints and } \\
\text { a higher percentage of CHOP-INTEND } \\
\text { response for secondary endpoints. }\end{array}$ \\
\hline
\end{tabular}

LP, lumbar puncture; CSF, cerebrospinal fluid; HFMSE, Hammersmith Functional Motor Scale-Expanded; HINE-2, Hammersmith Infant Neurological Exam-Part 2; CHOP-INTEND, Children's Hospital of Philadelphia Infant Test of Neuromuscular Disorders. 
to a 24-gauge or smaller needle. ${ }^{70}$

The PK data from the Phase 1 clinical trial were the same as those from other ODN clinical trials and animal studies. ${ }^{71}$ Concentrations of nusinersen were detectable more than 24 hours later in plasma, and 7 days later in CSF after dosing. In the $9 \mathrm{mg}$ group, $2.33 \pm 0.928 \mathrm{ng} / \mathrm{mL}$ was detected in CSF at 29 days after administration. Nusinersen was quickly taken up from the IT administration (CSF) site into circulation, and peak plasma levels emerged within a few hours after dosing. After IT dosing, plasma concentrations decreased slowly less than 20 hours after peaking, until being finally eliminated after 7 days. ${ }^{69}$

After the 9- to 14-month assessments, CSF nusinersen concentrations still maintained a quantified upper limit ( $1 \mathrm{ng} / \mathrm{mL})$. The terminal half-life of nusinersen in CNS tissues was $139 \pm 54$ days in monkeys, and 145-191 days in mice. The half-life in CSF was 132-166 days (4.5-6.0 months). Therefore, an infrequent nusinersen administration for maintaining drug levels can be achieved by this prolonged half-life, following drug loading of the target tissue. ${ }^{69} \mathrm{CSF}$ volume was similar in 2-year-old children based on the literature reporting that no obvious correlation was detected between CSF concentrations and age or total body weight. Therefore, it was appropriate that fixed doses of nusinersen were used in this pediatric population, and drug dose scaling was not necessary. ${ }^{72}$

The analysis of SMN protein levels of CSF samples suggested that variation did not occur in the $1 \mathrm{mg}$ or $3 \mathrm{mg}$ groups, while the levels of SMN protein were over double 9-14 months after dosing in the $6 \mathrm{mg}$ and $9 \mathrm{mg}$ groups compared to baseline. The clinical outcomes indicated that in the 1, 3, or 6 mg groups, there were no apparent changes in functional assay score evaluations from baseline at day 29, day 85, or 9-14 months after dosing. In contrast, improved functional assay scores were demonstrated in the 9 mg group at day 85 (3.1-point mean increase) from baseline, with further improvement at 9-14 months (5.8-point mean increase). Dose-limiting safety issues were not identified in this study, suggesting that testing doses of more than 9 mg should be taken into account. ${ }^{69}$

\section{CLINICAL PHASE 2 STUDIES OF NUSINERSEN}

Patients with homozygous gene mutations and deletions of SMN1, who had SMA originating from infancy, were used to assess the tolerability, PK, safety, and clinical efficacy by using multiple IT doses of nusinersen (equivalent to $6 \mathrm{mg}$ and $12 \mathrm{mg}$ doses) in an escalating dose clinical study of open-label Phase $2 .{ }^{73}$ Children aged between 3 weeks and 7 months old, of either gender, and with SMA symptoms occurring between 3 weeks and 6 months were recruited as eligible participants (Table 1). Loading doses of $6 \mathrm{mg}$ were received by the first four participants on days 1,15 , and 85 , following $12 \mathrm{mg}$ doses on day 253, and then every 4 months (6-12 mg group). The remaining 16 participants got $12 \mathrm{mg}$ equivalent doses according to the identical schedule (12 mg group). Follow-up visits on days 16, 29, $86,92,169,254,337$, and 442 were conducted, and then every 4 months. A follow-up of 9-32 months was implemented in the 6-12 mg group who received four to nine doses of nusinersen, while the follow-up of 2-27 months was executed in the $12 \mathrm{mg}$ group who received two to eight doses. Adverse events were experienced by all participants, with the most common being respiratory infections and respiratory distress or failure which are common in infantile SMA. The study drug was not or unlikely considered to be related to these common adverse events by investigators. ${ }^{73}$

Compared with baseline, 16 out of 19 participants (6-12 mg group with one of four participants; $12 \mathrm{mg}$ group with all 15 participants) were observed to have increased improvements in developmental motor milestones at the last visit based on motor milestones portion of the Hammersmith Infant Neurological Exam-Part 2 (HINE-2) ${ }^{74}$ From baseline to the last visit, there was a mean increment of 11.5 points on motor function overall ( $\mathrm{n}=18)$, and the Children's Hospital of Philadelphia Infant Test of Neuromuscular Disorders (CHOP-INTEND) ${ }^{75}$ was used to evaluate with improvement in 14 out of 18 infants. From baseline to the last visit, there was an increase in CHOP-INTEND in 12 out of 14 participants in the 12 mg group, compared to the infants with type I SMA who had a case series of natural history. Electrophysiological assessment using compound muscle action potentials (CMAP) suggested that compared to baseline, an increment in peroneal CMAP in all participants and ulnar CMAP in 12 of 15 patients were manifested in the $12 \mathrm{mg}$ group at last visit. As most participants were surviving without permanent ventilation, permanent ventilation or death in median age was not achieved. In the study, due to a case series of natural history used in comparison based on all the caveats, a differentiation in age at permanent ventilation or death was implicated by the log-rank Kaplan-Meier survival curve test. ${ }^{73}$

Nusinersen concentrations were determined by using electrochemiluminescence method. By analyzing the autopsy tissue of patients who received nusinersen, the drug was indicated to be taken up into glial cells throughout the CNS in the brainstem and other brain regions, and motor neurons throughout the vascular endothelial cells, neurons, and spinal cord. In all spinal cord areas, CSF drug concentrations were higher than $10 \mu \mathrm{g} / \mathrm{g}$ at 15-168 days after dosing. The infants with SMA who received nusinersen indicated that the SMN2 transcripts comprising exon 7 were $50-69 \%$ in thoracic cord tissues. By analyzing thoracic tissues of the spinal cord using a variation of reverse transcription-polymerase chain reaction and enzymelinked immunosorbent assay methods, ${ }^{76} 15-26 \%$ of the SMN2 transcripts consisted of exon 7 compared to infants with no disease and untreated infants with SMA. ${ }^{73}$ 


\section{CLINICAL PHASE 3 STUDIES OF NUSINERSEN}

A Phase 3 safety and efficacy trial (ENDEAR clinical trials) of nusinersen in infantile SMA were conducted, which were double-blind, randomized, and sham-controlled. ${ }^{77}$ In this study, 121 infants ( 7 months or less in age, sham control group vs. nusinersen group in a 1:2 ratio) who had experienced the assigned and randomization procedures were included in time-to-event analyses (Table 1). All of the infants were exposed to a dose that was equivalent to a $12 \mathrm{mg}$ dose in a 2-year-old person or older. A dose was delivered on days 1, 15, 29, and 64, and retention doses on days 183 and 302 were executed in the nusinersen group. Sham procedures were implemented on identical days in the control group. Assessment of the efficacy of endpoints was also performed on days $64,183,302$, and $394( \pm 7$ days at every visit). ${ }^{77}$

For primary endpoints, infants in the nusinersen group had a meaningfully higher percentage of motor-milestone response compared to those in the sham control group ( $41 \%$ vs. $0 \%$ ) in the interim analysis, indicating an early finish of the trial. Identically, infants in the nusinersen group had a significantly higher percentage of motor-milestone response than those in the control group ( $51 \%$ vs. $0 \%$ ) in the final analysis. The nusinersen group had a $47 \%$ lower incidence in the use of permanent assisted ventilation or the risk of death compared to the sham control group (hazard ratio $=0.53) .^{77}$

For secondary endpoints, infants in the nusinersen group had a significantly higher percentage of CHOP INTEND response than those in the control group. Meanwhile, infants in the nusinersen group had a lower percentage of mortality by the end of the trial than those in the control group (16\% vs. $39 \%$ ). The risk of death in the nusinersen group was $63 \%$ lower than that in the control group (hazard ratio=0.37). In subgroup analyses, the group with shorter disease duration at screening (the median duration $\leq 13$.1 weeks) was a higher likelihood of eventfree survival (time to use of permanent assisted ventilation or death) than the group with longer disease duration (the median duration $>13.1$ weeks), indicated that efficacy of nusinersen might be maximized by early initiation of treatment. ${ }^{77}$

Another nusinersen Phase 3 trial (CHERISH Clinical Trials) in 126 SMA children, with symptoms beginning at 6 months of age, was a sham-controlled, double-blinded, and multicenter trial. $^{78}$ The children (age ranging from 2-12 years, nusinersen group vs. sham control group in a 2:1 ratio) underwent the assigned and randomization procedures to have IT nusinersen administration at a dose of $12 \mathrm{mg}$ on days $1,29,85$, and $274 .^{78}$

In CHERISH, the total Hammersmith Functional Motor ScaleExpanded (HFMSE) ${ }^{79,80}$ score at month 15 of the least-squares mean change from baseline was used as the primary endpoint. An increase in total score indicated an enhancement of motor function, and a meaningful clinical results was defined as a variation of at least 3 points in the HFMSE score. The assess- ment of HFMSE score was implemented twice during the 4-week period of screening and at 3, 6, 9, 12, and 15 months by skilled clinical estimators. The study indicated that from baseline to month 15, the nusinersen group in HFMSE score had a least-squares mean increase (by 4.0 points), and the sham control group had a least-squares mean decrease (by -1.9 points) in the interim analysis, leading to an apparent difference between the two groups supporting nusinersen (least-squares mean difference in change $=5.9$ points). In the final analysis, the nusinersen group in HFMSE score had a least-squares mean increase from baseline to month 15 (by 3.9 points), and the sham control group had a least-squares mean decrease (by -1.0 points), resulting in a significant difference between the two groups supporting nusinersen (least-squares mean difference in change $=4.9$ point $){ }^{78}$

In the CHERISH study, the percentage of children increase from baseline in the HFMSE score was included as secondary endpoints, an outcome that implicates clinical enhancement of at least two motor skills. The study showed that there was a higher percentage of children in HFMSE score from baseline to month 15 in the nusinersen group, with an increase of at least three points ( $57 \%$ vs. $26 \%$ ), compared to the sham control group. $^{78}$

Being similar to Phase 1 and 2 studies, ${ }^{78}$ the incidence for total adverse events included procedural headache, procedural pain, back pain, procedural nausea, puncture syndrome, procedural nausea, the post-lumbar, nausea, headache, fluid leakage, vomiting, and cerebrospinal. LP was linked to the total rate of events, with very little difference between the control and the nusinersen groups. ${ }^{81,82}$

\section{CONCLUSION}

Due to the possibility of tissue damage and infection during injection, it may be challenging to use IT or ICV routes for CNS administration for chronic treatment, despite nusinersen having an infrequent dosing probability and a long half-life. Furthermore, the probability of complications arising from the use of long-term ICV or IT injections cannot be ruled out. The risks may be significantly reduced by an inhalation or oral administration method. However, in the course of any great endeavor to avoid the potential AEs in direct CNS administration and to find alternative delivery lines of effect oligonucleotides uptake of trans-BBB, the road is promising.

Furthermore, more studies are required to focus on extended characteristics of PD and PK of nusinersen on the CNS. To avoid liver absorption or kidney clearance, nusinersen with phosphonothioate backbones must remain intact in the blood and have extensive PK, which will possibly become more important to ensure more drugs stay in extra-hepatic tissues that have less receptors and a much slower receptor-mediated endocytosis rate. 
In the ENDEAR clinical trial, several symptomatic infants who had received nusinersen had not been cured, while some needed continued ventilatory support and feeding, some had not attained normal motor development, and a number of them had died. Therefore, like all promising therapeutic approaches, including small molecules, peptides, monoclonal antibodies, therapeutics, and virus vector-mediated gene delivery, ODN and RNA-based therapeutics also have limited ability in controlling disease progress since many diseases remain poorly understood.

In the future, new molecular pathogenic mechanisms of diseases should be studied in order to pose and develop more effective therapeutic approaches. Furthermore, chromosomal instability and gene mutations in disease may result from the body's cells reacting to stress stimulation from the outside world to retain the structural integrity of their own genes and proteins by increasing nucleotide number and gene diversity. It has been repurposed that transposon could alter genome size and be responsible for greater gene copy number. Therefore, a transposon-mediated gene-therapy strategy may be a practical approach in the future.

\section{ORCID iD}

Qing Li

https://orcid.org/0000-0001-5049-123X

\section{REFERENCES}

1. Hua Y, Sahashi K, Hung G, Rigo F, Passini MA, Bennett CF, et al. Antisense correction of SMN2 splicing in the CNS rescues necrosis in a type III SMA mouse model. Genes Dev 2010;24:1634-44.

2. Singh NK, Singh NN, Androphy EJ, Singh RN. Splicing of a critical exon of human Survival Motor Neuron is regulated by a unique silencer element located in the last intron. Mol Cell Biol 2006;26: 1333-46.

3. Wan L, Battle DJ, Yong J, Gubitz AK, Kolb SJ, Wang J, et al. The survival of motor neurons protein determines the capacity for snRNP assembly: biochemical deficiency in spinal muscular atrophy. Mol Cell Biol 2005;25:5543-51.

4. Hua Y, Vickers TA, Baker BF, Bennett CF, Krainer AR. Enhancement of SMN2 exon 7 inclusion by antisense oligonucleotides targeting the exon. PLoS Biol 2007;5:e73.

5. Kashima T, Manley JL. A negative element in SMN2 exon 7 inhibits splicing in spinal muscular atrophy. Nat Genet 2003;34:460-3.

6. Cartegni L, Krainer AR. Disruption of an SF2/ASF-dependent exonic splicing enhancer in SMN2 causes spinal muscular atrophy in the absence of SMN1. Nat Genet 2002;30:377-84.

7. Lunn MR, Wang CH. Spinal muscular atrophy. Lancet 2008;371: 2120-33.

8. Foust KD, Wang X, McGovern VL, Braun L, Bevan AK, Haidet AM, et al. Rescue of the spinal muscular atrophy phenotype in a mouse model by early postnatal delivery of SMN. Nat Biotechnol 2010;28: 271-4.

9. Coady TH, Shababi M, Tullis GE, Lorson CL. Restoration of SMN function: delivery of a trans-splicing RNA re-directs SMN2 premRNA splicing. Mol Ther 2007;15:1471-8.

10. Madocsai C, Lim SR, Geib T, Lam BJ, Hertel KJ. Correction of SMN2 Pre-mRNA splicing by antisense U7 small nuclear RNAs. Mol Ther
2005;12:1013-22.

11. Khorkova O, Wahlestedt C. Oligonucleotide therapies for disorders of the nervous system. Nat Biotechnol 2017;35:249-63.

12. Farr SA, Erickson MA, Niehoff ML, Banks WA, Morley JE. Central and peripheral administration of antisense oligonucleotide targeting amyloid- $\beta$ protein precursor improves learning and memory and reduces neuroinflammatory cytokines in Tg2576 (AßPPswe) mice. J Alzheimers Dis 2014;40:1005-16.

13. Juliano RL. The delivery of therapeutic oligonucleotides. Nucleic Acids Res 2016;44:6518-48.

14. Eckstein F. Nucleoside phosphorothioates. J Am Chem Soc 1970; 92:4718-23.

15. Bobst AM, Rottman F, Cerutti PA. Effect of the methylation of the 2 '-hydroxyl groups in polyadenylic acid on its structure in weakly acidic and neutral solutions and on its capability to form ordered complexes with polyuridylic acid. J Mol Biol 1969;46:221-34.

16. Evers MM, Toonen LJ, van Roon-Mom WM. Antisense oligonucleotides in therapy for neurodegenerative disorders. Adv Drug Deliv Rev 2015;87:90-103.

17. Hua Y, Vickers TA, Okunola HL, Bennett CF, Krainer AR. Antisense masking of an hnRNP A1/A2 intronic splicing silencer corrects SMN2 splicing in transgenic mice. Am J Hum Genet 2008;82:83448.

18. Rigo F, Hua Y, Krainer AR, Bennett CF. Antisense-based therapy for the treatment of spinal muscular atrophy. J Cell Biol 2012;199:21-5.

19. Juliano RL, Ming X, Nakagawa O. Cellular uptake and intracellular trafficking of antisense and siRNA oligonucleotides. Bioconjug Chem 2012;23:147-57.

20. Koller E, Vincent TM, Chappell A, De S, Manoharan M, Bennett CF. Mechanisms of single-stranded phosphorothioate modified antisense oligonucleotide accumulation in hepatocytes. Nucleic Acids Res 2011;39:4795-807.

21. Wagenaar TR, Tolstykh T, Shi C, Jiang L, Zhang J, Li Z, et al. Identification of the endosomal sorting complex required for transportI (ESCRT-I) as an important modulator of anti-miR uptake by cancer cells. Nucleic Acids Res 2015;43:1204-15.

22. Liang XH, Shen W, Sun H, Kinberger GA, Prakash TP, Nichols JG, et al. Hsp90 protein interacts with phosphorothioate oligonucleotides containing hydrophobic 2'-modifications and enhances antisense activity. Nucleic Acids Res 2016;44:3892-907.

23. Shemesh CS, Yu RZ, Gaus HJ, Seth PP, Swayze EE, Bennett FC, et al. Pharmacokinetic and pharmacodynamic investigations of ION353382, a model antisense oligonucleotide: using alpha-2-macroglobulin and murinoglobulin double-knockout mice. Nucleic Acid Ther 2016;26:223-35.

24. Shen W, Liang XH, Crooke ST. Phosphorothioate oligonucleotides can displace NEAT1 RNA and form nuclear paraspeckle-like structures. Nucleic Acids Res 2014;42:8648-62.

25. Liang XH, Shen W, Sun H, Prakash TP, Crooke ST. TCP1 complex proteins interact with phosphorothioate oligonucleotides and can co-localize in oligonucleotide-induced nuclear bodies in mammalian cells. Nucleic Acids Res 2014;42:7819-32.

26. Liang XH, Sun H, Shen W, Crooke ST. Identification and characterization of intracellular proteins that bind oligonucleotides with phosphorothioate linkages. Nucleic Acids Res 2015;43:2927-45.

27. Ezzat K, Helmfors H, Tudoran O, Juks C, Lindberg S, Padari K, et al. Scavenger receptor-mediated uptake of cell-penetrating peptide nanocomplexes with oligonucleotides. FASEB J 2012;26:117280.

28. Ugarte-Uribe B, Pérez-Rentero S, Lucas R, Aviñó A, Reina JJ, Alkorta I, et al. Synthesis, cell-surface binding, and cellular uptake of fluorescently labeled glucose-DNA conjugates with different carbohydrate presentation. Bioconjug Chem 2010;21:1280-7. 
29. Cheng CJ, Saltzman WM. Enhanced siRNA delivery into cells by exploiting the synergy between targeting ligands and cell-penetrating peptides. Biomaterials 2011;32:6194-203.

30. Alam MR, Dixit V, Kang H, Li ZB, Chen X, Trejo J, et al. Intracellular delivery of an anionic antisense oligonucleotide via receptor-mediated endocytosis. Nucleic Acids Res 2008;36:2764-76.

31. Juliano RL, Carver K, Cao C, Ming X. Receptors, endocytosis, and trafficking: the biological basis of targeted delivery of antisense and siRNA oligonucleotides. J Drug Target 2013;21:27-43.

32. Juliano RL, Ming X, Carver K, Laing B. Cellular uptake and intracellular trafficking of oligonucleotides: implications for oligonucleotide pharmacology. Nucleic Acid Ther 2014;24:101-13.

33. Sahay G, Querbes W, Alabi C, Eltoukhy A, Sarkar S, Zurenko C, et al. Efficiency of siRNA delivery by lipid nanoparticles is limited by endocytic recycling. Nat Biotechnol 2013;31:653-8.

34. Ming X, Alam MR, Fisher M, Yan Y, Chen X, Juliano RL. Intracellular delivery of an antisense oligonucleotide via endocytosis of a $\mathrm{G}$ protein-coupled receptor. Nucleic Acids Res 2010;38:6567-76.

35. Miller CM, Donner AJ, Blank EE, Egger AW, Kellar BM, Østergaard ME, et al. Stabilin-1 and Stabilin-2 are specific receptors for the cellular internalization of phosphorothioate-modified antisense oligonucleotides (ASOs) in the liver. Nucleic Acids Res 2016;44:278294.

36. Kortylewski M, Swiderski P, Herrmann A, Wang L, Kowolik C, Kujawski M, et al. In vivo delivery of siRNA to immune cells by conjugation to a TLR9 agonist enhances antitumor immune responses. Nat Biotechnol 2009;27:925-32.

37. Shi F, Sottile J. Caveolin-1-dependent betal integrin endocytosis is a critical regulator of fibronectin turnover. J Cell Sci 2008;121(Pt 14): 2360-71.

38. Juliano RL, Carver K. Cellular uptake and intracellular trafficking of oligonucleotides. Adv Drug Deliv Rev 2015;87:35-45.

39. Reyes-Reyes EM, Teng Y, Bates PJ. A new paradigm for aptamer therapeutic AS1411 action: uptake by macropinocytosis and its stimulation by a nucleolin-dependent mechanism. Cancer Res 2010;70:8617-29.

40. Ezzat K, Aoki Y, Koo T, McClorey G, Benner L, Coenen-Stass A, et al. Self-assembly into nanoparticles is essential for receptor mediated uptake of therapeutic antisense oligonucleotides. Nano Lett 2015;15:4364-73.

41. Kotula JW, Pratico ED, Ming X, Nakagawa O, Juliano RL, Sullenger BA. Aptamer-mediated delivery of splice-switching oligonucleotides to the nuclei of cancer cells. Nucleic Acid Ther 2012;22:187-95.

42. Wang S, Sun H, Tanowitz M, Liang XH, Crooke ST. Annexin A2 facilitates endocytic trafficking of antisense oligonucleotides. Nucleic Acids Res 2016;44:7314-30.

43. Prakash TP, Graham MJ, Yu J, Carty R, Low A, Chappell A, et al. Targeted delivery of antisense oligonucleotides to hepatocytes using triantennary $\mathrm{N}$-acetyl galactosamine improves potency 10 -fold in mice. Nucleic Acids Res 2014;42:8796-807.

44. Vickers TA, Crooke ST. Development of a quantitative BRET affinity assay for nucleic acid-protein interactions. PLoS One 2016;11: e0161930.

45. Castanotto D, Lin M, Kowolik C, Wang L, Ren XQ, Soifer HS, et al. A cytoplasmic pathway for gapmer antisense oligonucleotide-mediated gene silencing in mammalian cells. Nucleic Acids Res 2015; 43:9350-61.

46. Lorenz P, Baker BF, Bennett CF, Spector DL. Phosphorothioate antisense oligonucleotides induce the formation of nuclear bodies. Mol Biol Cell 1998;9:1007-23.

47. Kubo T, Zhelev Z, Bakalova R, Ohba H, Doi K, Fujii M. Controlled intracellular localization and enhanced antisense effect of oligonucleotides by chemical conjugation. Org Biomol Chem 2005;3:
3257-9.

48. Marcusson EG, Bhat B, Manoharan M, Bennett CF, Dean NM. Phosphorothioate oligodeoxyribonucleotides dissociate from cationic lipids before entering the nucleus. Nucleic Acids Res 1998; 26:2016-23.

49. Zelphati O, Szoka FC Jr. Mechanism of oligonucleotide release from cationic liposomes. Proc Natl Acad Sci U S A 1996;93:11493-8.

50. Naganuma T, Hirose T. Paraspeckle formation during the biogenesis of long non-coding RNAs. RNA Biol 2013;10:456-61.

51. Lorenz P, Misteli T, Baker BF, Bennett CF, Spector DL. Nucleocytoplasmic shuttling: a novel in vivo property of antisense phosphorothioate oligodeoxynucleotides. Nucleic Acids Res 2000;28:58292.

52. Doherty GJ, McMahon HT. Mechanisms of endocytosis. Annu Rev Biochem 2009;78:857-902.

53. Stein CA, Hansen JB, Lai J, Wu S, Voskresenskiy A, Høg A, et al. Efficient gene silencing by delivery of locked nucleic acid antisense oligonucleotides, unassisted by transfection reagents. Nucleic Acids Res 2010;38:e3.

54. Torres AG, Threlfall RN, Gait MJ. Potent and sustained cellular inhibition of miR-122 by lysine-derivatized peptide nucleic acids (PNA) and phosphorothioate locked nucleic acid (LNA)/2'-Omethyl (OMe) mixmer anti-miRs in the absence of transfection agents. Artif DNA PNA XNA 2011;2:71-8.

55. Smith RA, Miller TM, Yamanaka K, Monia BP, Condon TP, Hung $\mathrm{G}$, et al. Antisense oligonucleotide therapy for neurodegenerative disease. J Clin Invest 2006;116:2290-6.

56. Wahlestedt C, Golanov E, Yamamoto S, Yee F, Ericson H, Yoo H, et al. Antisense oligodeoxynucleotides to NMDA-R1 receptor channel protect cortical neurons from excitotoxicity and reduce focal ischaemic infarctions. Nature 1993;363:260-3.

57. Wahlestedt C, Pich EM, Koob GF, Yee F, Heilig M. Modulation of anxiety and neuropeptide Y-Y1 receptors by antisense oligodeoxynucleotides. Science 1993;259:528-31.

58. Wahlestedt C. Antisense oligonucleotide strategies in neuropharmacology. Trends Pharmacol Sci 1994;15:42-6.

59. Standifer KM, Chien CC, Wahlestedt C, Brown GP, Pasternak GW. Selective loss of delta opioid analgesia and binding by antisense oligodeoxynucleotides to a delta opioid receptor. Neuron 1994;12: 805-10.

60. Pechmann A, Langer T, Wider S, Kirschner J. Single-center experience with intrathecal administration of nusinersen in children with spinal muscular atrophy type 1. Eur J Paediatr Neurol 2018; 22:122-7.

61. Zanetta C, Nizzardo M, Simone C, Monguzzi E, Bresolin N, Comi GP, et al. Molecular therapeutic strategies for spinal muscular atrophies: current and future clinical trials. Clin Ther 2014;36:128-40.

62. Passini MA, Bu J, Richards AM, Kinnecom C, Sardi SP, Stanek LM, et al. Antisense oligonucleotides delivered to the mouse CNS ameliorate symptoms of severe spinal muscular atrophy. Sci Transl Med 2011;3:72ra18.

63. Geary RS. Antisense oligonucleotide pharmacokinetics and metabolism. Expert Opin Drug Metab Toxicol 2009;5:381-91.

64. Rigo F, Chun SJ, Norris DA, Hung G, Lee S, Matson J, et al. Pharmacology of a central nervous system delivered 2'-O-methoxyethylmodified survival of motor neuron splicing oligonucleotide in mice and nonhuman primates. J Pharmacol Exp Ther 2014;350:4655 .

65. Heemskerk H, de Winter C, van Kuik P, Heuvelmans N, Sabatelli P, Rimessi P, et al. Preclinical PK and PD studies on 2'-O-methylphosphorothioate RNA antisense oligonucleotides in the mdx mouse model. Mol Ther 2010;18:1210-7.

66. Verhaart IE, Tanganyika-de Winter CL, Karnaoukh TG, Kolfschoten 
IG, de Kimpe SJ, van Deutekom JC, et al. Dose-dependent pharmacokinetic profiles of 2'-O-methyl phosphorothioate antisense oligonucleotidesin mdx mice. Nucleic Acid Ther 2013;23:228-37.

67. Kordasiewicz HB, Stanek LM, Wancewicz EV, Mazur C, McAlonis MM, Pytel KA, et al. Sustained therapeutic reversal of Huntington's disease by transient repression of huntingtin synthesis. Neuron 2012;74:1031-44.

68. Geary RS, Norris D, Yu R, Bennett CF. Pharmacokinetics, biodistribution and cell uptake of antisense oligonucleotides. Adv Drug Deliv Rev 2015;87:46-51.

69. Chiriboga CA, Swoboda KJ, Darras BT, Iannaccone ST, Montes J, De Vivo DC, et al. Results from a phase 1 study of nusinersen (ISIS$\mathrm{SMN}(\mathrm{Rx})$ ) in children with spinal muscular atrophy. Neurology 2016;86:890-7.

70. Haché M, Swoboda KJ, Sethna N, Farrow-Gillespie A, Khandji, Xia $\mathrm{S}$, et al. Intrathecal injections in children with spinal muscular atrophy: nusinersen clinical trial experience. J Child Neurol 2016;31: 899-906.

71. Engelhardt JA. Comparative renal toxicopathology of antisense oligonucleotides. Nucleic Acid Ther 2016;26:199-209.

72. Matsuzawa J, Matsui M, Konishi T, Noguchi K, Gur RC, Bilker W, et al. Age-related volumetric changes of brain gray and white matter in healthy infants and children. Cereb Cortex 2001;11:335-42.

73. Finkel RS, Chiriboga CA, Vajsar J, Day JW, Montes J, De Vivo DC, et al. Treatment of infantile-onset spinal muscular atrophy with nusinersen: a phase 2, open-label, dose-escalation study. Lancet 2016; 388:3017-26.

74. Frisone MF, Mercuri E, Laroche S, Foglia C, Maalouf EF, Haataja L, et al. Prognostic value of the neurologic optimality score at 9 and 18 months in preterm infants born before 31 weeks' gestation. J
Pediatr 2002;140:57-60.

75. Glanzman AM, Mazzone E, Main M, Pelliccioni M, Wood J, Swoboda KJ, et al. The Children's Hospital of Philadelphia Infant Test of Neuromuscular Disorders (CHOP INTEND): test development and reliability. Neuromuscul Disord 2010;20:155-61.

76. Yu RZ, Baker B, Chappell A, Geary RS, Cheung E, Levin AA. Development of an ultrasensitive noncompetitive hybridization-ligation enzyme-linked immunosorbent assay for the determination of phosphorothioate oligodeoxynucleotide in plasma. Anal Biochem 2002;304:19-25.

77. Finkel RS, Mercuri E, Darras BT, Connolly AM, Kuntz NL, Kirschner $\mathrm{J}$, et al. Nusinersen versus sham control in infantile-onset spinal muscular atrophy. N Engl J Med 2017;377:1723-32.

78. Mercuri E, Darras BT, Chiriboga CA, Day JW, Campbell C, Connolly AM, et al. Nusinersen versus sham control in later-onset spinal muscular atrophy. N Engl J Med 2018;378:625-35.

79. Glanzman AM, O'Hagen JM, McDermott MP, Martens WB, Flickinger J, Riley S, et al. Validation of the Expanded Hammersmith Functional Motor Scale in spinal muscular atrophy type II and III. J Child Neurol 2011;26:1499-507.

80. O'Hagen JM, Glanzman AM, McDermott MP, Ryan PA, Flickinger J, Quigley J, et al. An expanded version of the Hammersmith Functional Motor Scale for SMA II and III patients. Neuromuscul Disord 2007;17:693-7.

81. Ebinger F, Kosel C, Pietz J, Rating D. Headache and backache after lumbar puncture in children and adolescents: a prospective study. Pediatrics 2004;113:1588-92.

82. Morgenlander JC. Lumbar puncture and CSF examination. Answers to three commonly asked questions. Postgrad Med 1994; 95:125-8. 